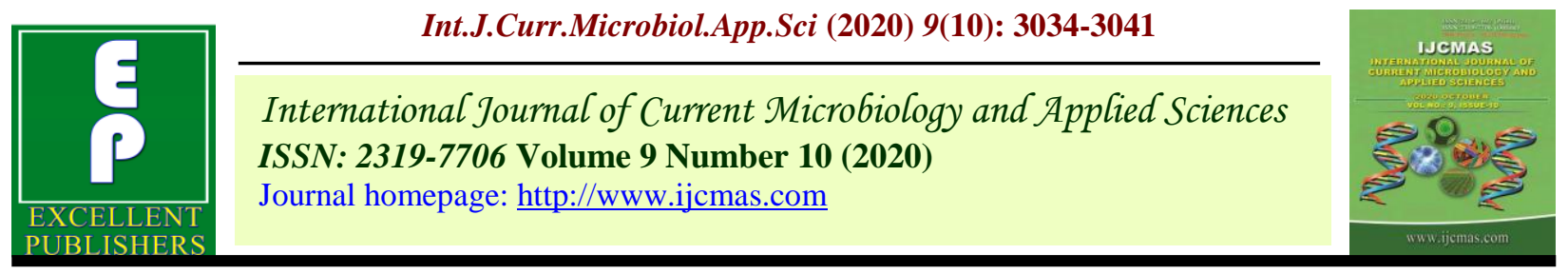

Original Research Article

https://doi.org/10.20546/ijcmas.2020.910.365

\title{
Evaluation of Pre and Post Emergent Herbicides in Kharif Groundnut (Arachis hypogaea L.) at Central Dry Zone of Karnataka, India
}

\author{
R. Venkateshwara ${ }^{\text {* }}$, A. H. Kumar Naik ${ }^{2}$, Parashuram Chandravamsi ${ }^{3}$, \\ T. Basavaraja Naik ${ }^{4}$ and M. S. Nandish ${ }^{5}$ \\ ${ }^{1}$ Dept. of Agronomy, ${ }^{4}$ Agronomy, ${ }^{5}$ Agril. Microbiology, College of Agriculture, \\ UAHS, Shivamogga, Karnataka, India \\ ${ }^{2}$ Agronomy, AICRP on Groundnut, ZAHRS, Hiriyur, Chitradurga Dist. Karnataka, India \\ ${ }^{3}$ Soil Science and Agril. Chemistry, College of Horticulture, Hiriyur, \\ Chitradurga Dist. Karnataka, India \\ *Corresponding author
}

\begin{abstract}
A B S T R A C T
Keywords

Weed,

Groundnut,

Herbicide

Article Info

Accepted:

24 September 2020

Available Online:

10 October 2020

Among the herbicide treatments, significantly lower total number of weeds (7.63, $6.12,7.84$ and $7.71 \mathrm{~m}^{-2}$ at $25,50,75 \mathrm{DAS}$ and at harvest, respectively) and total weed dry weight $\left(2.52,2.79,4.52\right.$ and $4.65 \mathrm{~g} \mathrm{~m}^{-2}$ at 25, 50, 75 DAS and at harvest, respectively) was recorded with treatment receiving Pendimethalin $30 \mathrm{EC}+$ Imazethapyr 2 EC @ $1.0 \mathrm{~kg} \mathrm{ha}^{-1} \mathrm{PE}$ (ready mix) + manual weeding at $30 \mathrm{DAS}$. Further the same treatment recorded significantly higher pod yield $\left(2,897 \mathrm{~kg} \mathrm{ha}^{-1}\right)$, kernel yield $\left(2,076 \mathrm{~kg} \mathrm{ha}^{-1}\right)$, haulm yield $\left(4,345 \mathrm{~kg} \mathrm{ha}^{-1}\right)$ and oil yield $\left(952 \mathrm{~kg} \mathrm{ha}^{-1}\right)$ and economic returns as compared to treatment with application of pendimethalin $38.7 \mathrm{CS} @ 1.0 \mathrm{~kg} \mathrm{ha}^{-1}$ pre-emergence.

\section{Introduction}

The average productivity of groundnut in India is about $1,436 \mathrm{~kg} \mathrm{ha}^{-1}$. In India, 70 per cent of the groundnut area and 75 per cent of the production is concentrated in the states of Gujarat, Tamil Nadu, Andhra Pradesh, Telangana, Maharashtra, Rajasthan and Karnataka. In Karnataka, groundnut is grown in an area of 6.56 lakh ha with a production of 5.83 lakh tonnes and productivity of $889 \mathrm{~kg}$ $\mathrm{ha}^{-1}$ (Anon. 2019). The major groundnut growing districts are Chitradurga, Dharwad,

Belagavi, Raichur, Vijayapura, Bellary and Bidar. The productivity of groundnut in these districts is lower as compared to national average productivity. Being a kharif crop and slow growth during initial stages, where groundnut encounters severe weed infestation problem especially in the early stages of their growth, as the crop seedling emerges seven to ten days after sowing coupled with the slow growth in the initial stages. The critical period for crop-weed competition in groundnut crop was reported to be, up to 45 DAS and by maintaining weed free condition during this
\end{abstract}


period results in higher pod yield (Geetha et $a l ., 2017)$. The weeds emerge faster and grow rapidly competing with the main crop for the resources namely soil moisture, nutrients, light and space. With the increasing labour scarcity, it is difficult to manage weeds by traditional method. Chemical weed management such as pre or post-emergent herbicide application gives a good result but couldn't control a broad spectrum of weeds. Hence combining the herbicides with manual weeding we can control a broad spectrum of weed population throughout the crop period. Pre-emergent herbicides prevent the germination of weed seeds in the beginning stages of the crop growth. Thus, there will be less weed infestation till the activity of herbicide present in the soil. An additional method to control weeds in later stages of crop growth is through the application of post-emergent herbicide which effectively controls matured weeds. So, post-emergent herbicides help in controlling the emerged weeds in later stages of groundnut crop where it is important to control the crop-weed competition at flowering and peg formation stages.

Keeping this in view, the present investigation entitled 'Evaluation of pre and post emergent herbicides for control of weeds in kharif groundnut (Arachis hypogaea L.)' was carried out at Central Dry Zone of Karnataka.

\section{Materials and Methods}

A field experiment was conducted during kharif season of 2019 at Zonal Agricultural and Horticultural Research Station, Hiriyur, Chitradurga district, Karnataka, to study the "Evaluation of pre and post emergent herbicides for control of weeds in kharif groundnut (Arachis hypogaea L.)". The experimental site situated between $13^{\circ} 57^{\prime} 32$ $\mathrm{N}$ latitude and $70^{\circ} 37^{\prime} 38 \mathrm{E}$ longitude with an altitude of 606.1 meters above mean sea level. It comes under Central Dry Zone (Agro- climatic zone 4) of Karnataka. The soil analysis data indicated that the soil was found to be alkaline in reaction with a $\mathrm{pH}$ of 8.1 and low in organic carbon $(0.47 \%)$. Further, the soil was low in available nitrogen, medium in phosphorus and potassium $(258,35$ and 315 $\mathrm{kg} \mathrm{ha}^{-1}$, respectively). G-2-52 a variety was sown in July with a spacing of $30 \mathrm{~cm} \times 10$ $\mathrm{cm}$. Experiment included nine treatments consisted of $\mathrm{T}_{1}$-Pendimethalin 38.7 CS @ 1.0 $\mathrm{kg} \quad \mathrm{ha}^{-1} \quad$ Pre-emergence (PE), $\mathrm{T}_{2^{-}}$ Pendimethalin $30 \mathrm{EC}+$ imazethapyr 2 EC @ $1.0 \mathrm{~kg} \mathrm{ha}^{-1} \mathrm{PE}$ (ready mix), $\mathrm{T}_{3}$ : Pendimethalin $38.7 \mathrm{CS} @ 1.0 \mathrm{~kg} \mathrm{ha}^{-1} \mathrm{PE}+$ quizalofop-pethyl $5 \%$ EC @ $50 \mathrm{~g} \mathrm{ha}^{-1}$ at 30 DAS Postemergence $(\mathrm{PoE}), \mathrm{T}_{4}$-Pendimethalin $30 \mathrm{EC}+$ imazethapyr 2 EC @ $1.0 \mathrm{~kg} \mathrm{ha}^{-1} \mathrm{PE}$ (ready mix) + quizalofop-p-ethyl $5 \%$ EC @ $50 \mathrm{~g}$

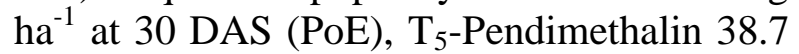
CS@1.0 kg ha ${ }^{-1} \mathrm{PE}+$ imazethapyr $10 \% \mathrm{SL}$ @ $75 \mathrm{~g} \mathrm{ha}^{-1}$ at 30 DAS, T6-Pendimethalin $38.7 \mathrm{CS} @ 1.0 \mathrm{~kg} \mathrm{ha}^{-1} \mathrm{PE}+$ manual weeding at 30 DAS, T 7 -Pendimethalin $30 \mathrm{EC} \mathrm{+}$ imazethapyr 2 EC @ $1.0 \mathrm{~kg} \mathrm{ha}^{-1}$ PE (ready mix $)+$ manual weeding at $30 \mathrm{DAS}, \mathrm{T}_{8^{-}}$ Intercultural operation at 15 and 30 DAS followed by manual weeding for intra rows and $\mathrm{T}_{9}$-Weedy check were laid out in Randomized Complete Block Design (RCBD) with three replications. Data on weed count and weed dry weight have shown high degree of variation. A relationship between the means and variance was observed. Therefore, the data on weed count and weed dry weight were subjected to $(\sqrt{(x+0.5})$ transformation to make analysis of variance more valid as suggested by Blackman and Roberts (1950). Standard statistical methods were used for comparing the treatment means.

\section{Results and Discussion}

\section{Weed density and dry weight}

Among the herbicide treatments, significantly lower total number of weeds $(7.63,6.12,7.84$ 
and $7.71 \mathrm{~m}^{-2}$ at 25, 50, 75 DAS and at harvest, respectively) (Table 1) and total weed dry weight $\left(2.52,2.79,4.52\right.$ and $4.65 \mathrm{~g} \mathrm{~m}^{-2}$ at 25 , 50,75 DAS and at harvest, respectively) (Table 2) was recorded with treatment receiving $\mathrm{T}_{7}$ : Pendimethalin $30 \mathrm{EC}+$ Imazethapyr 2 EC @ $1.0 \mathrm{~kg} \mathrm{ha}^{-1} \mathrm{PE}$ (ready mix) + manual weeding at 30 DAS over $T_{1}$ : Pendimethalin $38.7 \mathrm{CS} @ 1.0 \mathrm{~kg} \mathrm{ha}{ }^{-1} \mathrm{PE}$ $\left(10.39,10.88,11.07\right.$ and $11.25 \mathrm{~m}^{-2}$ at 25,50 , 75 DAS and at harvest) and (3.46, 4.64, 6.30 and $7.10 \mathrm{~g} \mathrm{~m}^{-2}$ at $25,50,75 \mathrm{DAS}$ and at harvest). The above results might be due to pre-emergent herbicide (ready mix) which prevents the emergence of grasses, sedges and broad-leaved weeds by inhibiting root and shoot growth so that there is less crop-weed competition at initial stages. According to Smita et al., (2014) revealed that presence of imazethapyr which is responsible for inhibition of acetolactate synthase (ALS) or actohydroxy acid synthase (AHAS) in broad leaved weeds which caused destruction of these weed growth. Similar observations were also made by Megha Dubey et al., (2010) and Kalaichelvi et al., (2015).

\section{Yield of groundnut}

Higher pod yield $\left(2,897 \mathrm{~kg} \mathrm{ha}^{-1}\right)$, kernel yield $\left(2,076 \mathrm{~kg} \mathrm{ha}^{-1}\right)$, haulm yield $\left(4,345 \mathrm{~kg} \mathrm{ha}^{-1}\right)$ and oil yield $\left(952 \mathrm{~kg} \mathrm{ha}^{-1}\right)$ at harvest recorded significantly higher in the treatment receiving $\mathrm{T}_{7}$ : Pendimethalin $30 \mathrm{EC}+$ Imazethapyr $2 \mathrm{EC}$ @ $1.0 \mathrm{~kg} \mathrm{ha}^{-1} \mathrm{PE}$ (ready mix) + manual weeding at 30 DAS. Significantly lower pod yield $\left(2,254 \mathrm{~kg} \mathrm{ha}^{-1}\right)$, kernel yield $(1,506 \mathrm{~kg}$ $\left.\mathrm{ha}^{-1}\right)$, haulm yield $\left(3,552 \mathrm{~kg} \mathrm{ha}^{-1}\right)$ and oil yield $\left(644 \mathrm{~kg} \mathrm{ha}^{-1}\right)$ at harvest recorded with single application $\mathrm{T}_{1}$ : Pendimethalin 38.7 CS @ 1.0 $\mathrm{kg} \mathrm{ha}^{-1}$ PE (Table 3). The reason for the increase in the number of pods per plant is due to lesser weed competition at initial stages which is controlled by the preemergent spray of pendimethalin which effect the cell division (microtubulaes assembly inhibitor) thus controls the germinated weed seeds (Aradhanabali et al., 2016) and one manual weeding at $30 \mathrm{DAS}$, helped in the loosening of soil which favours easy penetration and peg formation, thus increased the number of pods per plant. Similar results were also obtained by Kalhapure et al., (2013) and Jadhav et al., (2015).

\section{Economics}

The highest cost of cultivation incurred for plot receiving $\mathrm{T}_{8}$ : Intercultural operation at 15 and 30 DAS followed by manual weeding for intra rows (Rs. 58,315 $\mathrm{ha}^{-1}$ ) as compared to other treatments. Gross and net returns differed significantly due to different weed management practices. However, Intercultural operation at 15 and 30 DAS followed by manual weeding for intra rows significantly recorded higher gross returns (Rs. 1,37,720 $\mathrm{ha}^{-1}$ ) and net returns (Rs. 79,405 ha $\mathrm{ha}^{-1}$ ) over weedy check. However, it was on par with $\mathrm{T}_{7}$ : Pendimethalin 30 EC + Imazethapyr 2 EC @ $1.0 \mathrm{~kg} \mathrm{ha}^{-1} \mathrm{PE}$ (ready mix) + manual weeding at 30 DAS (Rs. 1,33,986 $\mathrm{ha}^{-1}$ and ₹ 78,7 $\mathrm{ha}^{-1}$, respectively) and $\mathrm{T}_{4}$ : Pendimethalin 30 $\mathrm{EC}+$ Imazethapyr $2 \mathrm{EC} @ 1.0 \mathrm{~kg} \mathrm{ha}^{-1} \mathrm{PE}$ (ready mix) + quizalofop-p-ethyl 5\% EC @ $50 \mathrm{~g} \mathrm{ha}^{-1}$ at 30 DAS (PoE) (Rs. 1,26,682 $\mathrm{ha}^{-1}$ and $73,378 \mathrm{ha}^{-1}$, respectively). Unlike observed in gross and net returns treatments receiving $\mathrm{T}_{7}$ : Pendimethalin $30 \mathrm{EC}+$ Imazethapyr 2 EC @ $1.0 \mathrm{~kg} \mathrm{ha}^{-1} \mathrm{PE}$ (ready mix) + manual weeding at 30 DAS (2.42) recorded significantly superior B: C over rest of the treatments. Where, weedy check culminated in lower B: C (0.92) (Table 4). This is due to the production of the higher pod and haulm yield by the effective control of weeds through an integrated method of weed management which effectively controlled weeds throughout the crop growth stage, because of the integrated method the cost of herbicide and labour was high which resulted in higher cost of cultivation. These findings are in line with these reported by Kumar et al., (2013) and Sardana et al., (2006). 
Table.1 Total number of weeds per $\mathrm{m}^{2}$ as influenced by weed management practices at different crop growth stages

\begin{tabular}{|c|c|c|c|c|c|}
\hline \multicolumn{2}{|r|}{ Treatments } & \multicolumn{4}{|c|}{ Total number of weeds } \\
\hline & & \multirow{2}{*}{$\begin{array}{c}25 \text { DAS } \\
10.39 * \\
(107.4)\end{array}$} & \multirow{2}{*}{$\begin{array}{c}\text { 50 DAS } \\
10.88 \\
(118.0)\end{array}$} & \multirow{2}{*}{$\begin{array}{c}75 \text { DAS } \\
11.07 \\
(122.0)\end{array}$} & \multirow{2}{*}{$\begin{array}{c}\text { At harvest } \\
11.25 \\
(126.2)\end{array}$} \\
\hline $\mathbf{T}_{1}$ & Pendimethalin 38.7 CS @ $1.0 \mathrm{~kg} \mathrm{ha}^{-1} \mathrm{PE}$ & & & & \\
\hline $\mathbf{T}_{2}$ & $\begin{array}{l}\text { Pendimethalin } 30 \mathrm{EC}+\text { Imazethapyr } 2 \mathrm{EC} @ 1.0 \mathrm{~kg} \mathrm{ha}^{-1} \mathrm{PE} \\
\text { (ready mix) }\end{array}$ & $8.16(66.1)$ & $\begin{array}{c}10.41 \\
(107.9)\end{array}$ & $\begin{array}{c}10.60 \\
(112.0)\end{array}$ & $\begin{array}{c}10.88 \\
(118.0)\end{array}$ \\
\hline $\mathbf{T}_{\mathbf{3}}$ & $\begin{array}{l}\text { Pendimethalin } 38.7 \mathrm{CS} @ 1.0 \mathrm{~kg} \mathrm{ha}^{-1} \mathrm{PE}+\text { quizalofop-p-ethyl } 5 \\
\% \text { EC @ } 50 \mathrm{~g} \mathrm{ha}^{-1} \text { at } 30 \mathrm{DAS}(\mathrm{PoE})\end{array}$ & $8.97(80.0)$ & $8.62(73.8)$ & $9.30(86.0)$ & $9.63(92.2)$ \\
\hline $\mathbf{T}_{4}$ & $\begin{array}{l}\text { Pendimethalin } 30 \mathrm{EC}+\text { Imazethapyr } 2 \mathrm{EC} @ 1.0 \mathrm{~kg} \mathrm{ha}^{-1} \mathrm{PE} \\
\text { (ready mix) + quizalofop-p-ethyl 5\% EC @ } 50 \mathrm{~g} \mathrm{ha}^{-1} \text { at } 30 \\
\text { DAS }(\mathrm{PoE})\end{array}$ & $7.95(62.8)$ & $8.20(66.7)$ & $8.63(74.0)$ & $8.69(75.0)$ \\
\hline $\mathbf{T}_{5}$ & $\begin{array}{l}\text { Pendimethalin } 38.7 \mathrm{CS} @ 1.0 \mathrm{~kg} \mathrm{ha}^{-1} \mathrm{PE}+\text { Imazethapyr } 10 \% \\
\text { SL @ } 75 \mathrm{~g} \mathrm{ha}^{-1}(\mathrm{PoE}) \text { at } 30 \mathrm{DAS}\end{array}$ & $9.25(85.1)$ & $8.83(77.4)$ & $9.64(92.5)$ & $9.69(93.5)$ \\
\hline $\mathbf{T}_{6}$ & $\begin{array}{l}\text { Pendimethalin } 38.7 \mathrm{CS} @ 1.0 \mathrm{~kg} \mathrm{ha}^{-1} \mathrm{PE}+\text { manual weeding at } \\
30 \mathrm{DAS}\end{array}$ & $9.17(83.6)$ & $6.61(43.2)$ & $8.31(68.6)$ & $8.47(71.2)$ \\
\hline $\mathbf{T}_{7}$ & $\begin{array}{l}\text { Pendimethalin } 30 \mathrm{EC}+\text { Imazethapyr } 2 \mathrm{EC} @ 1.0 \mathrm{~kg} \mathrm{ha}^{-1} \mathrm{PE} \\
\text { (ready mix })+ \text { manual weeding at } 30 \mathrm{DAS}\end{array}$ & $7.63(57.7)$ & $6.12(37.0)$ & $7.84(61.0)$ & $7.71(59.0)$ \\
\hline $\mathbf{T}_{8}$ & $\begin{array}{l}\text { Intercultural operation at } 15 \text { and } 30 \text { DAS followed by manual } \\
\text { weeding for intra rows }\end{array}$ & $3.94(15.0)$ & $5.37(28.3)$ & $6.10(36.7)$ & $5.86(33.8)$ \\
\hline $\mathbf{T}_{9}$ & Weedy check & $12.47(155.0)$ & $\begin{array}{c}12.88 \\
(165.4)\end{array}$ & $\begin{array}{c}13.16 \\
(172.6)\end{array}$ & $\begin{array}{c}12.71 \\
(161.1)\end{array}$ \\
\hline & S. Em \pm & 0.65 & 0.65 & 0.72 & 0.71 \\
\hline & CD at $5 \%$ & 1.95 & 1.96 & 2.17 & 2.13 \\
\hline
\end{tabular}

Note: PE: Pre-emergence (2 DAS), PoE: Post-emergence (30 DAS), DAS: Days after sowing, SL: Soluble liquid, EC: Emulsifiable concentration, CS: Capsule suspension, * - Transformed values $(\sqrt{x+0.5})$, figures in the parentheses indicate original values. 
Table. 2 Total weeds dry weight per $\mathrm{m}^{2}$ as influenced by weed management practices at different crop growth stages

\begin{tabular}{|c|c|c|c|c|c|}
\hline \multicolumn{2}{|r|}{ Treatments } & \multicolumn{4}{|c|}{ Total weeds dry weight $\left(\mathrm{g} \mathrm{m}^{-2}\right)$} \\
\hline & & \multirow{2}{*}{$\begin{array}{c}25 \text { DAS } \\
\left.3.46^{*} 11.5\right)\end{array}$} & \multirow{2}{*}{$\begin{array}{c}50 \text { DAS } \\
4.64(21.0)\end{array}$} & \multirow{2}{*}{$\begin{array}{c}75 \text { DAS } \\
6.30(39.2)\end{array}$} & \multirow{2}{*}{$\begin{array}{c}\begin{array}{c}\text { At } \\
\text { harvest }\end{array} \\
7.10(49.9)\end{array}$} \\
\hline $\mathbf{T}_{1}$ & Pendimethalin38.7 CS @ $1.0 \mathrm{~kg} \mathrm{ha}^{-1} \mathrm{PE}$ & & & & \\
\hline $\mathbf{T}_{2}$ & Pendimethalin $30 \mathrm{EC}+$ Imazethapyr $2 \mathrm{EC} @ 1.0 \mathrm{~kg} \mathrm{ha}^{-1} \mathrm{PE}$ (ready mix) & $2.80(7.4)$ & $4.31(18.1)$ & $6.00(35.5)$ & $6.54(42.3)$ \\
\hline $\mathbf{T}_{3}$ & $\begin{array}{l}\text { Pendimethalin } 38.7 \mathrm{CS} @ 1.0 \mathrm{~kg} \mathrm{ha}^{-1} \mathrm{PE}+\text { quizalofop-p-ethyl } 5 \% \text { EC } \\
@ \quad 50 \mathrm{~g} \mathrm{ha}^{-1} \text { at } 30 \mathrm{DAS}(\mathrm{PoE})\end{array}$ & $3.39(11.0)$ & $4.02(15.7)$ & $5.50(29.7)$ & $5.51(29.9)$ \\
\hline $\mathbf{T}_{4}$ & $\begin{array}{l}\text { Pendimethalin } 30 \mathrm{EC}+\text { Imazethapyr } 2 \mathrm{EC} @ 1.0 \mathrm{~kg} \mathrm{ha}^{-1} \mathrm{PE} \text { (ready mix) } \\
\text { + quizalofop-p-ethyl } 5 \% \mathrm{EC} @ 50 \mathrm{~g} \mathrm{ha}^{-1} \text { at } 30 \mathrm{DAS}(\mathrm{PoE})\end{array}$ & $2.57(6.1)$ & $3.82(14.1)$ & $4.87(23.2)$ & $5.12(25.7)$ \\
\hline $\mathbf{T}_{5}$ & $\begin{array}{l}\text { Pendimethalin } 38.7 \mathrm{CS} @ 1.0 \mathrm{~kg} \mathrm{ha}^{-1} \mathrm{PE}+\text { Imazethapyr } 10 \% \mathrm{SL} @ \\
75 \mathrm{~g} \mathrm{ha}^{-1}(\mathrm{PoE}) \text { at } 30 \mathrm{DAS}\end{array}$ & $3.38(10.9)$ & $4.09(16.3)$ & $6.06(36.2)$ & $6.07(36.4)$ \\
\hline $\mathbf{T}_{6}$ & Pendimethalin38.7 CS @ $1.0 \mathrm{~kg} \mathrm{ha}^{-1} \mathrm{PE}+$ manual weeding at $30 \mathrm{DAS}$ & $3.32(10.5)$ & $3.88(14.5)$ & $5.23(26.9)$ & $5.06(25.1)$ \\
\hline $\mathbf{T}_{7}$ & $\begin{array}{l}\text { Pendimethalin } 30 \mathrm{EC}+\text { Imazethapyr } 2 \mathrm{EC} @ 1.0 \mathrm{~kg} \mathrm{ha}^{-1} \mathrm{PE} \text { (ready mix) } \\
\text { + manual weeding at } 30 \mathrm{DAS}\end{array}$ & $2.52(5.8)$ & $2.79(7.3)$ & $4.52(19.9)$ & $4.65(21.1)$ \\
\hline $\mathbf{T}_{8}$ & $\begin{array}{l}\text { Intercultural operation at } 15 \text { and } 30 \text { DAS followed by manual weeding } \\
\text { for intra rows }\end{array}$ & $1.81(2.8)$ & $2.16(4.2)$ & 3.66 (12.9) & $3.16(9.5)$ \\
\hline $\mathbf{T}_{9}$ & Weedy check & $6.0(35.5)$ & $7.29(52.6)$ & $8.21(66.9)$ & $9.21(84.4)$ \\
\hline & S. Em \pm & 0.3 & 0.3 & 0.4 & 0.4 \\
\hline & CD at $5 \%$ & 0.8 & 0.9 & 1.2 & 1.3 \\
\hline
\end{tabular}

Note: PE: Pre-emergence (2 DAS), PoE: Post-emergence (30 DAS), DAS: Days after sowing, SL: Soluble liquid, EC: Emulsifiable concentration, CS: Capsule suspension, * - Transformed values $(\sqrt{x+0.5})$, figures in the parentheses indicate original values 
Table.3 Yield parameters of groundnut as influenced by weed management practices

\begin{tabular}{|c|c|c|c|c|c|}
\hline \multicolumn{2}{|r|}{ Treatments } & \multicolumn{4}{|c|}{ Yield parameters } \\
\hline & & \multirow{2}{*}{$\begin{array}{c}\text { Pod yield } \\
\left.\text { (kg ha }^{-1}\right)\end{array}$} & \multirow{2}{*}{$\begin{array}{c}\begin{array}{c}\text { Kernel } \\
\text { yield } \\
\left.\text { (kg ha }^{-\mathbf{1}}\right)\end{array} \\
1506\end{array}$} & \multirow{2}{*}{$\begin{array}{c}\begin{array}{c}\text { Haulm } \\
\text { yield (kg } \\
\left.\text { ha }^{-1}\right)\end{array} \\
3552\end{array}$} & \multirow{2}{*}{$\begin{array}{c}\begin{array}{c}\text { Oil yield } \\
\left.\text { (kg ha }^{-1}\right)\end{array} \\
644\end{array}$} \\
\hline $\mathbf{T}_{1}$ & Pendimethalin $38.7 \mathrm{CS} @ 1.0 \mathrm{~kg} \mathrm{ha}^{-1} \mathrm{PE}$ & & & & \\
\hline $\mathbf{T}_{2}$ & $\begin{array}{l}\text { Pendimethalin } 30 \mathrm{EC}+\text { Imazethapyr } 2 \mathrm{EC} @ 1.0 \mathrm{~kg} \mathrm{ha}^{-1} \mathrm{PE} \text { (ready } \\
\text { mix) }\end{array}$ & 2333 & 1618 & 3710 & 696 \\
\hline $\mathbf{T}_{\mathbf{3}}$ & $\begin{array}{l}\text { Pendimethalin } 38.7 \mathrm{CS} @ 1.0 \mathrm{~kg} \mathrm{ha}^{-1} \mathrm{PE}+\text { quizalofop-p-ethyl } 5 \% \text { EC } \\
@ 50 \mathrm{~g} \mathrm{ha}^{-1} \text { at } 30 \mathrm{DAS}(\mathrm{PoE})\end{array}$ & 2504 & 1803 & 4048 & 781 \\
\hline $\mathbf{T}_{4}$ & $\begin{array}{l}\text { Pendimethalin } 30 \mathrm{EC}+\text { Imazethapyr } 2 \mathrm{EC} @ 1.0 \mathrm{~kg} \mathrm{ha}^{-1} \mathrm{PE} \text { (ready } \\
\text { mix) + quizalofop-p-ethyl } 5 \% \mathrm{EC} @ 50 \mathrm{~g} \mathrm{ha}^{-1} \text { at } 30 \mathrm{DAS}(\mathrm{PoE})\end{array}$ & 2738 & 1909 & 4206 & 861 \\
\hline $\mathbf{T}_{5}$ & $\begin{array}{l}\text { Pendimethalin } 38.7 \mathrm{CS} @ 1.0 \mathrm{~kg} \mathrm{ha}^{-1} \mathrm{PE}+\text { Imazethapyr } 10 \% \mathrm{SL} @ \\
75 \mathrm{~g} \mathrm{ha}^{-1}(\mathrm{PoE}) \text { at } 30 \mathrm{DAS}\end{array}$ & 2487 & 1786 & 3925 & 799 \\
\hline $\mathbf{T}_{6}$ & Pendimethalin38.7 CS @ $1.0 \mathrm{~kg} \mathrm{ha}^{-1} \mathrm{PE}+$ manual weeding at $30 \mathrm{DAS}$ & 2540 & 1829 & 4167 & 805 \\
\hline $\mathbf{T}_{7}$ & $\begin{array}{l}\text { Pendimethalin } 30 \mathrm{EC}+\text { Imazethapyr } 2 \mathrm{EC} @ 1.0 \mathrm{~kg} \mathrm{ha}^{-1} \mathrm{PE} \text { (ready } \\
\text { mix) + manual weeding at } 30 \mathrm{DAS}\end{array}$ & 2897 & 2076 & 4345 & 952 \\
\hline $\mathbf{T}_{8}$ & $\begin{array}{l}\text { Intercultural operation at } 15 \text { and } 30 \text { DAS followed by manual weeding } \\
\text { for intra rows }\end{array}$ & 2976 & 2135 & 4623 & 982 \\
\hline $\mathbf{T}_{9}$ & Weedy check & 975 & 586 & 2053 & 242 \\
\hline & S. Em \pm & 165 & 125.9 & 281 & 56 \\
\hline & CD at $5 \%$ & 494 & 377 & 844 & 168 \\
\hline
\end{tabular}

Note: PE: Pre-emergence (2 DAS), PoE: Post-emergence (30 DAS), DAS: Days after sowing, EC: Emulsifiable concentration, CS: Capsule suspension, SL: Soluble liquid. 
Table.4 Economics of groundnut as influenced by different weed management practices

\begin{tabular}{|c|c|c|c|c|c|}
\hline \multicolumn{2}{|r|}{ Treatments } & \multirow{2}{*}{$\begin{array}{c}\begin{array}{c}\text { Cost of } \\
\text { cultivation } \\
\left(₹ \text { ha }^{-1}\right)\end{array} \\
52165\end{array}$} & \multirow{2}{*}{$\begin{array}{c}\begin{array}{c}\text { Gross } \\
\text { returns } \\
\left.\text { (₹ ha }^{-1}\right)\end{array} \\
104333 \\
\end{array}$} & \multirow{2}{*}{$\begin{array}{c}\begin{array}{c}\text { Net } \\
\text { returns } \\
\left(₹ \text { ha }^{-1}\right)\end{array} \\
52168\end{array}$} & \multirow{2}{*}{$\begin{array}{l}\text { B: C } \\
2.00\end{array}$} \\
\hline $\mathbf{T}_{1}$ & Pendimethalin38.7 CS @1.0 kg ha ${ }^{-1} \mathrm{PE}$ & & & & \\
\hline $\mathbf{T}_{2}$ & $\begin{array}{l}\text { Pendimethalin } 30 \mathrm{EC}+\text { Imazethapyr } 2 \mathrm{EC} @ 1.0 \mathrm{~kg} \mathrm{ha}^{-1} \mathrm{PE} \\
\text { (ready mix) }\end{array}$ & 52205 & 108007 & 55802 & 2.07 \\
\hline $\mathbf{T}_{\mathbf{3}}$ & $\begin{array}{l}\text { Pendimethalin } 38.7 \mathrm{CS} @ 1.0 \mathrm{~kg} \mathrm{ha}^{-1} \mathrm{PE}+\text { quizalofop-p-ethyl } 5 \\
\% \text { EC @ } 50 \mathrm{~g} \mathrm{ha}^{-1} \text { at } 30 \mathrm{DAS}(\mathrm{PoE})\end{array}$ & 53255 & 115956 & 62701 & 2.18 \\
\hline $\mathbf{T}_{4}$ & $\begin{array}{l}\text { Pendimethalin } 30 \mathrm{EC}+\text { Imazethapyr } 2 \mathrm{EC} @ 1.0 \mathrm{~kg} \mathrm{ha}^{-1} \mathrm{PE} \\
\text { (ready mix) + quizalofop-p-ethyl } 5 \% \mathrm{EC} @ 50 \mathrm{~g} \mathrm{ha}^{-1} \text { at } 30 \mathrm{DAS} \\
(\mathrm{PoE})\end{array}$ & 53305 & 126682 & 73378 & 2.38 \\
\hline $\mathbf{T}_{5}$ & $\begin{array}{l}\text { Pendimethalin } 38.7 \mathrm{CS} @ 1.0 \mathrm{~kg} \mathrm{ha}^{-1} \mathrm{PE}+\text { Imazethapyr } 10 \% \mathrm{SL} \\
@ 75 \mathrm{~g} \mathrm{ha}^{-1}(\mathrm{PoE}) \text { at } 30 \mathrm{DAS}\end{array}$ & 53015 & 115121 & 62106 & 2.17 \\
\hline $\mathbf{T}_{6}$ & $\begin{array}{l}\text { Pendimethalin } 38.7 \mathrm{CS} @ 1.0 \mathrm{~kg} \mathrm{ha}^{-1} \mathrm{PE}+\text { manual weeding at } 30 \\
\text { DAS }\end{array}$ & 52415 & 117653 & 65239 & 2.24 \\
\hline $\mathbf{T}_{7}$ & $\begin{array}{l}\text { Pendimethalin } 30 \mathrm{EC}+\text { Imazethapyr } 2 \mathrm{EC} @ 1.0 \mathrm{~kg} \mathrm{ha}^{-1} \mathrm{PE} \\
\text { (ready mix })+ \text { manual weeding at } 30 \mathrm{DAS}\end{array}$ & 55255 & 133986 & 78732 & 2.42 \\
\hline $\mathbf{T}_{8}$ & $\begin{array}{l}\text { Intercultural operation at } 15 \text { and } 30 \text { DAS followed by manual } \\
\text { weeding for intra rows }\end{array}$ & 58315 & 137720 & 79405 & 2.36 \\
\hline $\mathbf{T}_{9}$ & Weedy check & 49315 & 45389 & -3925 & 0.92 \\
\hline & S. Em \pm & - & 5872 & 2301 & 0.09 \\
\hline & CD at $5 \%$ & - & 17606 & 6900 & 0.26 \\
\hline
\end{tabular}

Note: PE: Pre-emergence (2 DAS), PoE: Post-emergence (30 DAS), DAS: Days after sowing, EC: Emulsifiable concentration, CS: Capsule suspension, SL: Soluble liquid. 


\section{References}

Anonymous, 2019, Directorate of economics and statistics, https://www.indiastat. com.

Aradhana., Bali, Bazaya, B. R., Lekh, Chand and Sanjay, Swami, 2016. Weed management in Soybean (Glycine max L.). Int. J. Life Sci., 11(1): 255-257.

Blackman.G.E. and Roberts, H.A., 1950. Studies on selective weed control. Agril. Sci., 40: 62-69.

Geetha, D. S., Venkateshwarulu, S. and Chandrasekar, K., 2017. Effect of integrated weed management practices on weed dynamics, yield and economics of Rabi groundnut (Arachis hypogaea) in sandy loam soils of Andhra Pradesh, Int. J. Curr. Res., 9(01): 44605-44608.

Jadhav, P. B., Singh, R., and Kamble, D. R., 2015. Effect of weed control methods on growth and yield of groundnut. Adv. Res. J. Crop Improv., 6(2): 151-157.

Kalaichelvi, K., Sakthivel, S. and Balakrishnan, A., 2015. Integrated weed management in groundnut. Indian J. Weed Sci., 47(2): 174 -177.

Kalhapure, A. H., Shete, B. T. and Bodake, P. S. 2013. Integration of chemical and cultural methods for weed management in groundnut. Indian J. Weed Sci., 45(2): 116-119.

Kumar, Y., Saxena, R., Gupta, K. C., Fagaria, V. D. and Singh, R., 2013. Yield attributes and yield of groundnut (Arachis hypogaea L.) as influenced by weed management practices in semiarid region. J. Crop Weed., 9(2): 185189.

Megha Dubey., Smita Singh., Kewat, M. L. and Sharma, J. K., 2010. Efficacy of imazethapyr against weeds in groundnut. Indian J. Weed Sci., 42(1\&2): 27-30.

Sardana, V., Walia, U. S. and Kandhola, S. S., 2006. Productivity and economics of summer groundnut (Arachis hypogaea L.) cultivation as influenced by weed management practices. Indian J. Weed Sci., 18(1\&2): 156-158.

Smita, Singh, Kewat, M. L., Megha, Dubey, Shukla, U. N. and Sharma, J. K., 2014. Efficacy of imazethapyr on weed dynamics, yield potential and economics of groundnut (Arachis hypogaea L.). Int. J. Legume Res., 37(1): 87-92.

\section{How to cite this article:}

Venkateshwara, R., A. H. Kumar Naik, Parashuram Chandravamsi, T. Basavaraja Naik and Nandish, M. S. 2020. Evaluation of Pre and Post Emergent Herbicides in Kharif Groundnut (Arachis hypogaea L.) at Central Dry Zone of Karnataka, India. Int.J.Curr.Microbiol.App.Sci. 9(10): 3034-3041. doi: https://doi.org/10.20546/ijcmas.2020.910.365 